\title{
Designing and Evaluating a Guiding and Positioning System for Indoor Navigation
}

\author{
Yuan WANG*, Weidong HUANG, and Henry Been-Lirn DUH \\ Human Interface Technology Laboratory Australia \\ University of Tasmania, Newnham, Australia \\ ${ }^{*}$ Corresponding author
}

Keywords: Wireless Sensor Networks, RFID, Ubiquitous and Pervasion Computing, Internet of Things, Location and Handoff Management.

\begin{abstract}
Mobile devices such as smart phones have gained their popularity for the purpose of information acquisition in recent years. In order to provide users with the information in indoor environments, several indoor mobile guiding systems have been developed. Most of the systems focus on how to offer qualified indoor navigation services, mainly based on geographical data and analyses. However, only having a navigation service seems to be insufficient and inefficient. Thus applications aimed at providing comprehensive information for visitors in indoor environments are needed. In this paper, we describe the design and implementation of an indoor guiding and positioning system, which not only enables a navigation service but also provides multiple types of information automatically about the indoor environments when users enter an area of the environment. Furthermore, an iPhone application, InSchool, is developed to evaluate the performance of this designed system and the test results indicate significant enhancement to positional accuracy of the application.
\end{abstract}

\section{Introduction}

The Internet of Things (IoT) is being touted as the next technological revolution [1]. With the rapid growth of IoT, technologies like RFID, wireless communications, real-time localization, and sensor networks make it into a reality. The number of sensors and the communication technologies that are integrated into smart phones are increasing such as accelerometer, gyroscope, Wi-Fi and Bluetooth [2][20]. All of these features have given mobile phone users advanced capability to access information about their personal contexts (location, movement and surroundings). These personal contexts could imply the information and services that a user may request [3] especially for a visitor who is not familiar with the building that he is going to enter. Much research has been conducted to demonstrate the usefulness of location information for improvement of visiting experience [4][5][6][21]. Although a large body of research can be found in the literature concerning the technologies of indoor positioning, there are only a few papers discussing how to use location information to benefit users.

Another issue that is worth further exploration is the design differences of user interfaces for outdoor environments and indoor environments. Generally speaking, according to our prototype design survey, users expect more information and more interactions during indoor visits due to their intentional entrance, which means they request information more than just for indoor navigation. These demands lead to system designers to provide more details, such as what is the function of one room, which area 
has activities now and the introduction of people in this building or what people are doing here.

In an attempt to address these issues, we designed and developed an indoor navigation system. This system is a smart phone based application. It collects location information from pre-distributed devices called iBeacon, which in turn is used to retrieve corresponding information from the cloud server to present relevant information to the mobile user.

\section{Related Work}

\section{Review of Mobile Input Methods}

The interface design is affected by how information is entered, while interface features could determine how users could access the information [17]. The common mobile phone input methods can be summarized as below:

- $\quad$ Bar, button, hyperlinks to press;

- $\quad$ List, menus, tab navigation, to choose from;

- Text field and search bar to type in;

- Breadcrumbs to track;

- Interactive map and virtual 3D guide to explore;

- Voice and touchscreen gesture to recognize;

- Wearable device and sensors to detect;

Most input methods and interfaces are expected to be intuitive for good user experience. But considering the tasks given to participants in past usability tests, there are always some situations in which users feel hard to operate or to find what they want. The original purpose of offering input methods is to perceive users' request and to leave flexibility for users to decide. However, if users do not know which type of information they could access for, they may try different inputs and keep exploring the interface.

Another point mentioned by Kenteris et al. [18] is that all input methods inevitably divert visitors' attentions from the real visit, although they aim at assisting. Under this circumstance, audio input could solve this partly. But the negative impact still exists because of the potential noise output.

Most researchers regard users' location information as a type of context rather than a method of input. If users could obtain different interfaces to demonstrate ample information at different locations, then they may be able to keep their attentions focused as well as save the time needed for input. Therefore, their visit experience could be enhanced. Taking advantage of the problem-solving strategy mentioned by Sung et al. [19] and this automatic working method, users could find interesting events that have not been visited by themselves with the proximity notification.

\section{Review of Existing Mobile Guide Systems}

Many mobile guiding systems are developed for outdoor environments [7][8][9]. They could offer a route suggestion and estimate the travel time after choosing a destination. On the basis of these outdoor mobile guide systems, several prototypes have been presented as mobile indoor guidance [10][11]. However, the functions and services of these indoor mobile guiding systems are highly similar to outdoor ones. The main difference is the positioning technology that is adopted. For outdoor environments, GPS is widely used, while for indoor environments, Wi-Fi, Bluetooth, RFID and location mark recognition are mostly used. 
Bluetooth, Wi-Fi, and RFID are adopted for RSSI to transmit its signal and then calculate the location [12]. These three approaches can be classified as base station positioning [13]. The non-RSSI technologies such as multi-camera tracking and inertial navigation positioning are also used. The multi-camera tracking technology is based on the cooperation of multiple cameras, and can also be realized by using wearable devices [14]. The core point is to detect the features in each single frame of each single camera. The inertial navigation system (INS) is 'old', compared with other technologies mentioned above. It was originally designed for adjusting a rocket's flying posture by the American rocket pioneer Robert Goddard with the explanation of rudimentary gyroscopic systems [15]. An INS makes use of motion sensors (such as accelerometers) and rotation sensors (such as gyroscopes) to calculate a moving object's position, orientation, or velocity (the speed in a given direction) without the demand of any other equipment [16].

Several commercial indoor guiding systems such as Meridian (http:// meridianapps.com/), Quantitec (http: //quantitec.de/), Infsoft (http: //www.infsoft.com/) and Wifarer (http://www.wifarer.com/) have been developed to provide more information in addition to navigation to meet indoor user requirements. The additional information consists of visiting plan, 3-D map, current location and indoor location analytics. These services could be applied among different indoor environments including museums, airports, hotels, university campuses, and so on. They developed these general features prior to expanding their application venues widely.

\section{System Overview}

In an attempt to address the previously mentioned challenges, we proposed a mobile guiding system with a well-defined framework. The goal for the system is to not only enable a navigation service but also provide multiple types of information automatically about the indoor environment that users enter into. The system was designed to support the indoor scenario, which is expected to have an open Wi-Fi or a cellular network access, in order for users to retrieve information needed from the server.

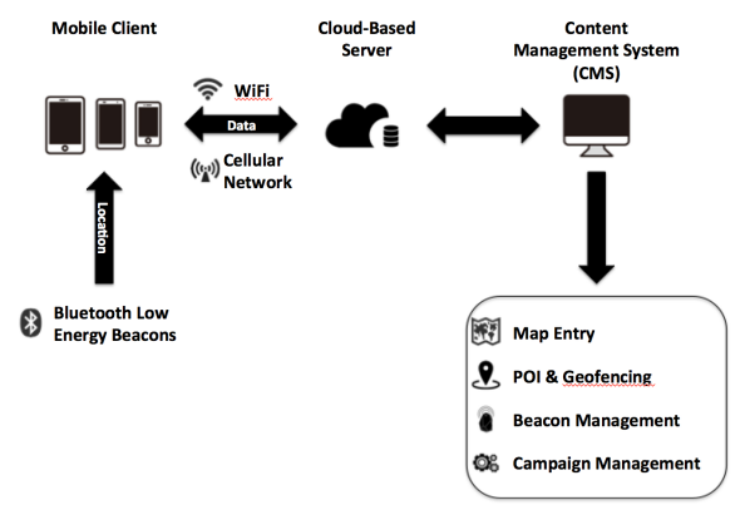

Figure 1. System framework.

The overview of the proposed system is shown in Figure 1.Taking advantage of Bluetooth low energy technology (BLE 4.0), the iBeacon base station can broadcast a universally unique identifier (UUID) to nearby devices with a settled frequency. And then the devices, such as smartphones, can calculate the distance by using RSSI. The space around one base station is divided into four regions with three boundaries. When 
users enter or exit one boundary, an event is triggered with the UUID of the beacon and the identified boundary. This event can be a push notification and an application interface from the device itself or other network storage servers. Therefore, the developed prototype system consists of a number of iBeacon base stations, a mobile application and a respective cloud-based server.

As shown in Figure 2, the concept design of a mobile client for this system is to give visitors the ability to receive information without any input. Meanwhile, all the information will be classified and selected so that visitors can have a general understanding of the indoor environment quickly.
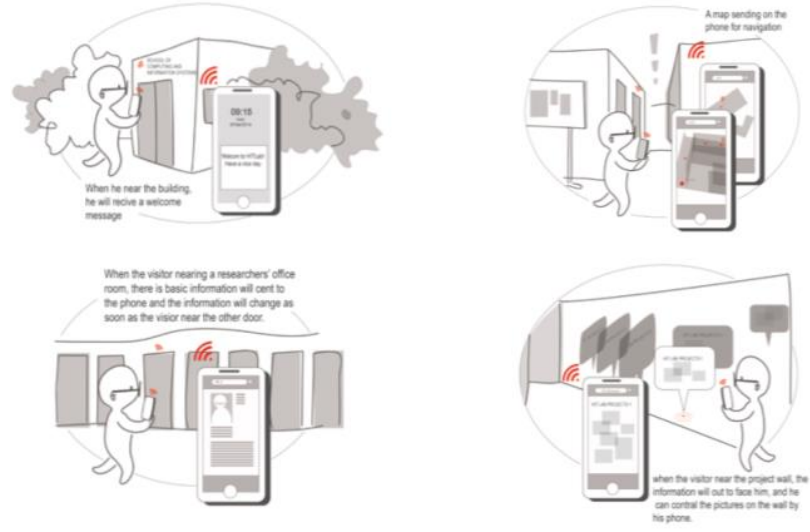

Figure 2. Mobile client concept design.

\section{Two Design Choices about Indoor Map}

The indoor map is a key factor of indoor mobile guiding systems. The interactions that users are required to make and the amount of information provided for users to access on the map determine the quality of the whole system to some extent. Two map features are discussed in sub-sections below.
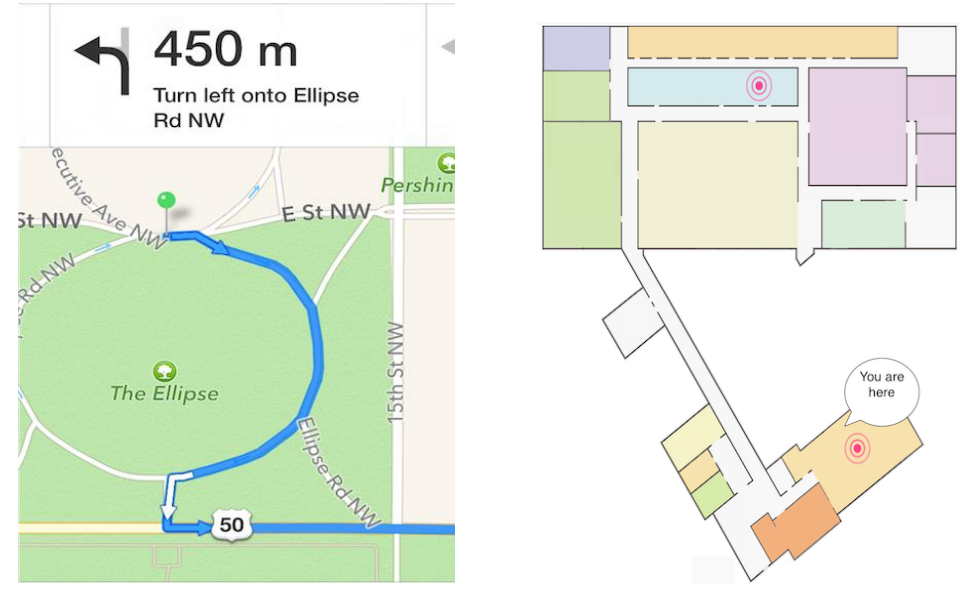

Figure 3. Screenshots of routes provided by Google Maps mobile application (left); an example of an indoor map (right)

Route Navigation: Route navigation is a classic service for outdoor mobile guiding system as shown in Figure 3 (left). There is usually some distance between the starting point and the destination with multiple choices of routes. Meanwhile, the information of route turnings may be complex. As a result, most mobile interfaces do not show the 
destination on the screen. Instead, they focus on a 'turn by turn' feature to guide users and try to enrich the details of surrounding area. Figure 3 (right) shows a typical indoor map of a school building. Maps of most school buildings only cover a limited area if compared with general outdoor environments. Therefore, users could see both of their current location as well as their chosen destination on one mobile phone screen, while the map proportion is still sufficient to recognize the route without other instructions. Additionally, the door position of the destination room could be useful for users to plan their routes.

Based on the above discussion, if an indoor mobile guiding system is developed specifically for school building visitors in order for users to easily find their way with marks of current location and destination, offering the service of route navigation may not be necessary. Showing more information at same time may also bring potential distraction and it is meaningful to keep only most necessary features and simplify the map.

Zooming: Zooming is one significant interaction type with map, which actually adjusts the amount of information that is provided. However, a practical issue for zooming is that it cannot indicate how much users should zoom to access the information they want. Users have to keep zooming if they fail to obtain that. This will happen with a higher possibility if users want to select a destination, which is far away from the current location. In this paper, the system we are going to design is for school building visitors. Here we propose a new concept of hierarchical map switching instead of map zooming. The basic idea of it is to offer several area maps with different degrees of details; the area maps could be switched from one to another by users. This interaction may save users' operation time and allow them to access the details of target area faster without considering how much zoom they should operate.

\section{System Implementation}

In this paper, we particularly focus on a map aided navigation solution, which is developed for indoor environments and try to provide information needed for covering different prospects. Our design is based on an initial hybrid location. By matching with a floor plan database, users will receive a positional accuracy. In order to demonstrate the feasibility and accuracy, indoor tests were conducted with an iPhone demo application named InSchool, which is specifically designed for visitors who are going to visit school buildings at the School of Computing \& Information System (CIS) in the University of Tasmania. It can provide information of entering notification, indoor map, current location, laboratory introduction, staff members' profile, projects demonstration, seminar schedule and contacts (when leaving the building).

This application was developed with four core components: system registration, indoor positioning, information provision and location awareness interface.

\section{System Registration}

A counter is used to record the visit history. If users enter one area or room where they have come before, a notification with button will be generated. Meanwhile, related information will be hidden unless the button is pressed. We also add a manual operation mode as a supplement of automatic information provision. Therefore, if users have special needs to access information out of the building, they could switch to the manual mode. 


\section{Indoor Positioning}

iBeacon is adopted to measure the distance between a user and Bluetooth base stations. Then trigonometry is adopted to locate the user's position. In order to reduce the interference of Bluetooth signals from the building users, these base stations are placed underneath the roof of the building.

Three parameters are used to optimize the calculation result: the height of the iBeacons, the scale coefficient between the distance on the map and the real value and the compensation coefficient for each iBeacon.

\section{Information Provision}

Taking advantage of the positioning system, users could receive information on mobile phones automatically without any input (in fact, we use their locations as an uninterrupted input). Meanwhile, if they exit an area, information will disappear automatically as well. For example, when users come to the entrance of school, they could get the new events list; when users come to the seminar room, they could get the seminar schedules; when users come to a laboratory space, they could get the project demos developed by both academic staff members and students; when users leave the CIS building, the time they spent in each area will be shown as well as the school contacts, and so on. In this way, users have no need to search about the room nearby. Therefore, they can focus more on the visit itself. The screenshots of several interfaces are shown in Figure 4.
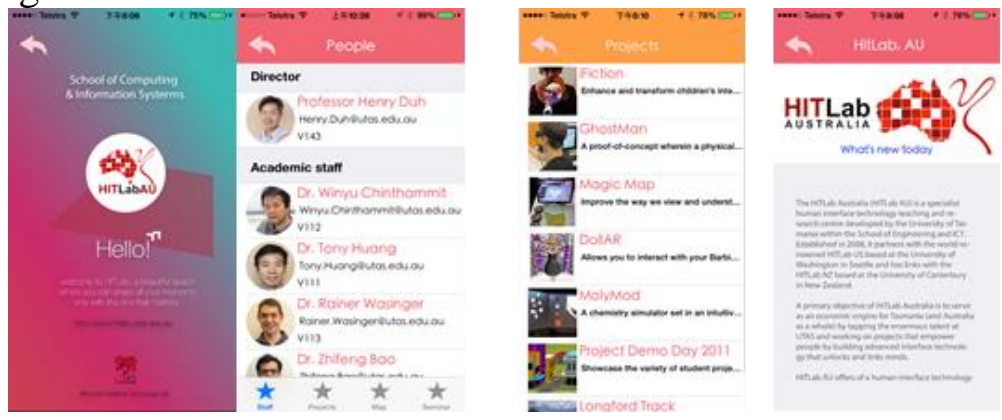

Figure 4. Screenshots of several interfaces that will be triggered.

\section{Location Awareness Interface}
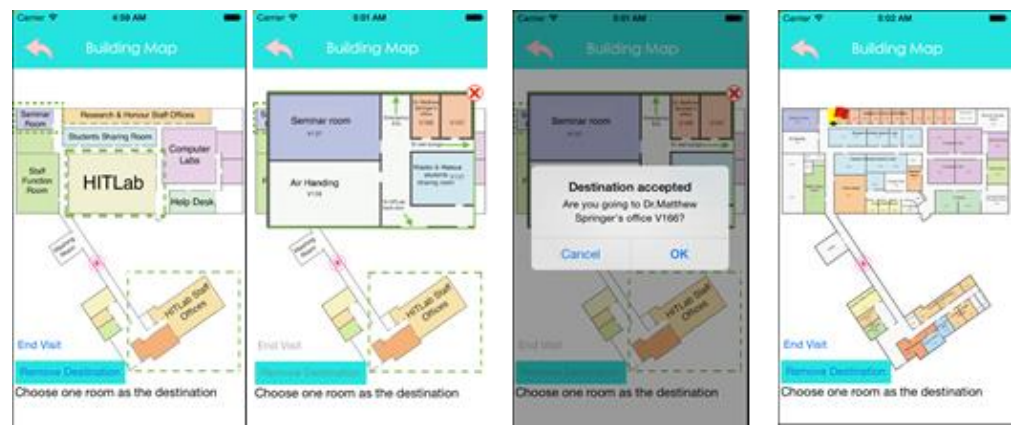

Figure 5. Screenshots of hierarchical map switch.

In order to show users' current location and the map information as well, the hierarchical maps are designed, which are presented in Figure 5. The whole indoor map is divided into several areas based on their functions, while the twinkling boundaries indicate the areas in which there are events that are currently on. This kind of simplified maps may help the user to get the core information on the map quickly. A detailed sub-map will appear if one area is pressed and the user can continue to choose one room 
for its particular information and mark it as a destination. After this, the room will be highlighted with a flag to indicate the position of the door. The room area will keep flashing until the user arrives. One feature here is that the automatic information provision still works during this map interaction. This ensure that the user will not miss anything interesting on his way.

\section{System Evaluation}

A prototype of the system has been developed based on the design. To test the functionality and usability of the prototype, we conducted two preliminary evaluations, which we describe the sub-sections below.

\section{Functionality Test}

The developed algorithms were field tested in indoor environments and yielded accurate matching results as well as a significant enhancement to positional accuracy. There were 500 samples taken for the distance test from the phone to one iBeacon. The average value of the total of these samples was 3.425, which is close to the real distance of 3.5. Another test was conducted to get knowledge about the distance measurements when the user is moving. In this test three iBeacons were placed, and the tester took one mobile phone from a settled starting point to a settled end point. The distances between the user and the three beacons were recorded along with the user's movements. All the five tests presented the same routines.

\section{Usability Test}

The usability test was conducted in a university building. This building was chosen as its inside layout was complex enough and required carful navigation to find target location: some rooms were close to each other, while others were further away. These rooms were used for different purposes, such as offices, seminar rooms, laboratories, classrooms, and meeting rooms. And getting into these rooms require a navigation of a long corridor with a number of turns. iBeacons were distributed and configured to location awareness.

A total of ten participants were recruited to make a trial of the InSchool application. They were all mobile phone frequent users and none of them were familiar with the test building. Each participant was given an iPhone 5 with the system being pre-installed in it. They were required to complete a set of tasks based on a visiting scenario in which they were asked to navigate the building to seek specific information from specific locations of the building. They were asked to explore the whole building as visitors and each was given a different scenario, but with the similar level of difficulty and complexity. This would allow us to test the usability of the full functionality of the system. The time they took to complete the tasks was recorded.

At the end, participants were asked to complete a questionnaire about their visit experience and the system performance. Out of the fifteen questions, three were about the personal information, one about the usage history of mobile guiding systems, seven about the service quality, three about the interface quality and one about the open idea. Additionally, five participants were interviewed individually to comment on whether the application was easy to learn and easy to use. All the interviews were recorded for transcripts.

The quantitative analysis for the questionnaires and the qualitative analysis for interviews are still in process. However, the initial feedback we have received was 
positive. All participants were able to complete their assigned tasks within a reasonable time. The automatic provision feature got a score of 3.9. The hierarchical map switch got a score of 3.8 and the total application got a score of 4.0. The range of rating score used in the tests was between 1 and 5, in which 5 means the most helpful and 1 means the most helpless.

There were five questions asking participants to rate the system service from 0 to 5 , where five means that a user is totally satisfied and zero means totally dissatisfied. The five key features that were asked to be rated included automatic information receiving, area awareness, easy to organize the route, helpful to visit and the total performance. The user ratings for these features were 4.1, 4.2, 3.8, 3.8 and 3.9 respectively.

\section{Conclusion}

The achieved results demonstrated that the proposed map aided navigation system using the building information and the map matching algorithm with a BLE technology is useful. It enhances the reliability, usability, and accuracy of navigation trajectories in indoor environments.

We have presented a mobile indoor guiding system that can provide users with positioning and navigation services. Compared to that with outdoor environments, the information that users may request in indoor environments is more specific and detailed. This paper discussed the need for the interaction and information types for mobile guiding systems. Then an automatic information provision method and the hierarchical map switch interface were presented. Although our demo application is designed for school visitors, its service can be expanded to other indoor environments such as airports, hotels and office buildings.

\section{Acknowledgment}

The authors would like to thank the Sense-T Project for sponsoring this research work.

\section{References}

[1] L. Trappeniers, M. A. Feki, F. Kawsar, and M. Boussard, "The internet of things: the next technological revolution," Computer, vol. 46, no. 2, pp. 0024-25, 2013.

[2] J. Shang, S. Yu, F. Gu, Z. Xu, and L. Zhu, “A mobile guide system framework for museums based on local location-aware approach." Computer Science and Service System (CSSS), 2011 International Conference on, 2011. IEEE, 1935-1940.

[3] W.-P. Lee, "Deploying personalized mobile services in an agent-based environment," Expert Systems with Applications, vol. 32, no. 4, pp. 1194-1207, 2007.

[4] M. Petrie, and L. Tallon, "The iPhone effect? Comparing visitors' and museum professionals' evolving expectations of mobile interpretation tools." 2010.

[5] J. Lanir, A. J. Wecker, T. Kuflik, E. Dim, I. Beja, I. Belinky, I. Gordon, N. Kashtan, I. Mogilevsky, and M. Zancanaro, "From a Research Prototype to a Deployed System: the Case of the Hecht Museum Visitor's Guide." p. 53, 2010.

[6] T.-H. Do and M. Yoo. "TDOA-based indoor positioning using visible light." Photonic Netw. Commun. 27, 2 (April 2014), 80-88, 2014. 
[7] J. C. Ching, C. Domingo, K. Iglesia, C. Ngo, and N. Chua, "Mobile indoor positioning using Wi-Fi localization and image processing," Theory and Practice of Computation, pp. 242-256: Springer, 2013.

[8] L. Klingbeil, M. Romanovas, P. Schneider, M. Traechtler, and Y. Manoli, "A modular and mobile system for indoor localization." pp. 1-10, 2010.

[9] T. S.-H. Wang, D. Tjondronegoro, M. Docherty, W. Song, and J. Fuglsang, "A recommendation for designing mobile pedestrian navigation system in university campuses." pp. 3-12, 2013.

[10]H. F. El-Sofany, and S. A. El-Seoud, "Mobile Tourist Guide-An Intelligent Wireless System to Improve Tourism, using Semantic Web," iJIM, vol. 5, no. 4, pp. 4-10, 2011.

[11]J. A. B. Link, P. Smith, N. Viol, and K. Wehrle, "Footpath: Accurate map-based indoor navigation using smartphones." pp. 1-8, 2011.

[12] J. Neidig "Algorithms Increase Operational Reliability of UHF RFID Systems," RFID Journal, pp. 1-5, March, 2013.

[13]R. Li, B. Zhang, S. S. Sundar, and H. B.-L. Duh, "Interacting with Augmented Reality: how does location-based AR enhance learning?," Human-Computer Interaction-INTERACT 2013, pp. 616-623: Springer, 2013.

[14]Y. Tian, W. R. Hamel, and J. Tan, "Accurate human navigation using wearable monocular visual and inertial sensors," Instrumentation and Measurement, IEEE Transactions on, vol. 63, no. 1, pp. 203-213, 2014.

[15]R. H. Battin, "Space guidance evolution-a personal narrative," Journal of Guidance, Control, and Dynamics, vol. 5, no. 2, pp. 97-110, 1982.

[16]X. Yin, Y. Sun, and C. Wang, "Positioning errors predicting method of strapdown inertial navigation systems based on PSO-SVM." 2013

[17]C. Emmanouilidis, R. Koutsiamanis, A. Tasidou, "Mobile guides: Taxonomy of architectures, context awareness, technologies and applications". Journal of Network and Computer Applications, 36, 1(2013), 103-125.

[18]M. Kenteris, D. Gavalas, D. Economou, "Electronic mobile guides: a survey". Personal and ubiquitous computing, 15, 1 (2011), 97-111.

[19] Y.-T. Sung, H.-T. Hou, C.-K. Liu, K.-E. Chang. "Mobile guide system using problem-solving strategy for museum learning: a sequential learning behavioural pattern analysis". Journal of computer assisted learning, 26, 2 (2010), 106-115.

[20]L. Alem, W. Huang, "Recent trends of mobile collaborative augmented reality systems", Springer Science \& Business Media, 2011.

[21] W. Huang, L. Alem, "A usability and spatial awareness study of near-eye displays". Proc. 2012 IEEE International Conference on Systems, Man, and Cybernetics (SMC). IEEE, Seoul; 2012:906-911. 